NUC-MINN-96/15-T

December 1996

\title{
COULOMB EFFECTS ON CHARGED KAON DISTRIBUTIONS FROM VLASOV DYNAMICS
}

\author{
Alejandro Ayala ${ }^{\dagger}$ \\ Department of Physics \\ University of Illinois \\ Urbana, IL 61801-3080 \\ Joseph Kapusta \\ School of Physics and Astronomy \\ University of Minnesota \\ Minneapolis, MN 55455
}

\begin{abstract}
We compute the influence of Coulomb effects on the charged kaon distribution at low momentum in high energy nucleus-nucleus collisions. This is accomplished by solving the Vlasov equation for the kaons in the presence of an expanding, highly charged, fireball.
\end{abstract}

† ayala1@rsm1.physics.uiuc.edu

$\ddagger$ kapusta@physics.spa.umn.edu 
The main goal of colliding heavy nuclei at high energy is to produce high density, high temperature matter, either hadronic or quark-gluon plasma. One way of studying the properties of high energy density matter is to compare the single-particle momentum distributions to those in a nucleon-nucleon collision at the same beam energy. The many-body medium should be most influential on the low momentum, or long deBroglie wavelength, part of the spectra and least influential on the high momentum, or short deBroglie wavelength, part. For example, there was a preliminary report of an enhancement of the charged kaon distributions in the low momentum region as compared to nucleon-nucleon collisions in an experiment at Brookhaven National Laboratory's AGS [1]. Although this enhancement is now known to have been caused by an albedo source at the spectrometer's collimator edge close to the beam trajectory [2], it begs the question of how the enormous electrical charge in a central gold-gold collision $(Z=158)$ modifies the low momentum spectra of charged hadrons.

The most widely used approach to incorporate Coulomb effects into the description of the charged particle distributions is by means of the Gamow factor [3].

$$
G(\eta)=|\psi(r=0)|^{2}=\left|\frac{2 \pi \eta}{e^{2 \pi \eta}-1}\right|
$$

Here $\eta=Z_{1} Z_{2} \alpha / v, v$ is the relative speed of the particle (charge $Z_{1}$ ) with the source (charge $Z_{2}$ ), and $\psi(r=0)$ is the relative Coulomb wave function evaluated at zero separation between the two (point) charges. In this approach, the charged particle distribution is given in terms of the neutral particle distribution and $G$ by

$$
\frac{d^{3} N^{\mathrm{ch}}(p)}{d p^{3}} \approx \frac{d^{3} N^{0}(p+k(p))}{d p^{3}} \times G(\eta)
$$

where $k(p)$ is the shift in the momentum due to the Coulomb kick.

The original motivation for the introduction of eq. (11) is the similarity to barrier penetration in fission and because this formula arises in a perturbative calculation to first order in $Z \alpha$ for low momenta. But for heavy ion reactions, where the total charge $Z$ is such that $Z \alpha \sim 1$, a first order perturbative approach to Coulomb effects is likely to fail and so will an approach that mimics such. Figure 1 shows the neutral, positive, and negative kaon momentum distributions in the fireball rest frame obtained from eq. (2) using a MaxwellBoltzmann distribution for neutrals and a non-expanding fireball with charge $Z=158$ and kaon temperature $T=120 \mathrm{MeV}$. The neutral distribution is normalized to unity. Generally the Gamow-modifed charge distributions do not preserve the normalization so the areas under those curves are not 1 . Figure 2 shows the exact classical result obtained from the formula

$$
\frac{d N}{d E}=\int d^{3} r d^{3} v f\left(\mathbf{r}, \mathbf{v}, t_{0}\right) \delta\left(E-m v^{2} / 2-V_{C}(r)\right)
$$


where $V_{C}(r)$ is the Coulomb potential felt by the kaon. The initial phase space distribution $f$ is taken to be a Maxwell-Boltzmann equilibrium distribution

$$
\left.f \mathbf{r}, \mathbf{v}, t_{0}\right) \propto \exp \left\{-\left[m v^{2} / 2+V_{C}(r)\right] / T\right\} \Theta\left(R_{0}-r\right)
$$

which constrains the kaons to lie within a uniform density charged fireball of radius $R_{0}=8$ $\mathrm{fm}$. The differences between the two figures are quite apparent and indicates the extent to which the Gamow-modified neutral spectrum can be relied on.

A more important physical effect which the Gamow factor (1) does not take into account at all is that in a high energy heavy ion collision the matter blows apart, and in fact it is the low energy detected charged particle which is left behind.

Therefore it is desirable to look for an alternative approach to the description of Coulomb effects on charged particle spectra in high energy heavy ion collisions. In this paper we explore the influence of a time-dependent electric field produced by an expanding charged fireball on the low momentum spectrum of a test charge particle. To be suitable as a test particle the hadron must not be produced in great abundance and its nonelectromagnetic interaction with other hadrons at low relative momentum must not be very strong, otherwise it will influence the expansion of the fireball and the problem must be solved self-consistently. Kaons are good test particles at AGS type energies. For simplicity and definiteness we assume a thermal momentum distribution at the strong interaction freeze out and dynamically evolve this distribution by means of the Vlasov equation. The interaction of a single test particle with the rest of the matter is purely electromagnetic. Since the particles that produce the electric field are moving away from the collision region, the external field is time dependent. We describe the motion of nonrelativistic test particles, thus ignoring interactions with the magnetic component of the electromagnetic field. We model the expanding fireball as a uniformly charged sphere, as viewed from the center-of-mass of the colliding nuclei, with a radius growing linearly with time. This expansion model is motivated by the similarity solution to a hydrodynamically expanding fireball [⿴囗十. This model turns out to be solvable analytically albeit in terms of Bessel functions. A serious comparison with experiment may require a simulation based on an event generator like ARC [5] or RQMD [6]. Finally, we remark that a description in terms of classical equations of motion for the test charge should be adequate for kaons with kinetic energy greater than about $10 \mathrm{MeV}$. At this energy the kaon DeBroglie wavelength is $2 \mathrm{fm}$ which is small enough compared to expected gradients of temperature and density in the fireball.

A uniformly charged sphere which has a radius $R_{0}$ at time $t_{0}$, whose total charge is $Z e$, and whose radius $R$ is increasing with time at a constant speed $v_{s}$ produces an electric potential

$$
V(r, t)=\left\{\begin{array}{ll}
Z e / 4 \pi r, & r \geq R=v_{s} t \\
Z e\left(3 R^{2}-r^{2}\right) / 8 \pi R^{3}, & r \leq R=v_{s} t
\end{array} .\right.
$$

The fireball parameters are not independent but are related by $R_{0}=v_{s} t_{0}$. We first concentrate on the interior $\left(r \leq v_{s} t\right)$ potential. If $f^{ \pm}(\mathbf{r}, \mathbf{v}, t)$ represents the $\pm e$ charge 
particle phase space distribution then, when ignoring particle collisions after decoupling, its dynamics is governed by Vlasov's equation. In the interior region

$$
\left[\frac{\partial}{\partial t}+\mathbf{v} \cdot \frac{\partial}{\partial \mathbf{r}} \pm \frac{t_{s}}{4 t^{3}} \mathbf{r} \cdot \frac{\partial}{\partial \mathbf{v}}\right] f^{ \pm}(\mathbf{r}, \mathbf{v}, t)=0
$$

where

$$
t_{s} \equiv \frac{Z e^{2}}{\pi m v_{s}^{3}},
$$

$m$ is the kaon's mass, and $t \geq t_{0}$. Notice that $c$, the speed of light, does not enter into the definition of the characteristic time $t_{s}$ since we work in the non-relativistic limit.

The solution to eq. (6) is found by the method of characteristics. This involves solving the classical equations of motion and using the solutions to evolve the initial distribution $f^{ \pm}\left(\mathbf{r}, \mathbf{v}, t_{0}\right)$ forward in time.

In the interior the equations of motion are

$$
\begin{aligned}
& \frac{d \mathbf{r}}{d t}=\mathbf{v} \\
& \frac{d \mathbf{v}}{d t}= \pm \frac{t_{s}}{4 t^{3}} \mathbf{r}
\end{aligned}
$$

or equivalently

$$
\frac{d^{2} \mathbf{r}}{d t^{2}}= \pm \frac{t_{s}}{4 t^{3}} \mathbf{r}
$$

For the initial distribution we take a Maxwell-Boltzmann equilibrium distribution at temperature $T$.

$$
f^{ \pm}\left(\mathbf{r}, \mathbf{v}, t_{0}\right) \propto \exp \left\{-\left[m v^{2} / 2 \pm e V\left(r, t_{0}\right)\right] / T\right\} \Theta\left(R_{0}-r\right)
$$

Note that this constrains the kaons to lie within the initial sphere.

The solution to the equations of motion for positive kaons is

$$
\mathbf{r}(t)=\mathbf{A} \sqrt{t} I_{1}\left(\sqrt{t_{s} / t}\right)+\mathbf{B} \sqrt{t} K_{1}\left(\sqrt{t_{s} / t}\right)
$$

and for negative kaons is

$$
\mathbf{r}(t)=\mathbf{A} \sqrt{t} J_{1}\left(\sqrt{t_{s} / t}\right)+\mathbf{B} \sqrt{t} N_{1}\left(\sqrt{t_{s} / t}\right)
$$

where the constant vectors $\mathbf{A}$ and $\mathbf{B}$ are determined by the initial conditions. The solution to eq. (6) incorporating the initial condition is

$$
\begin{aligned}
f^{ \pm}(\mathbf{r}, \mathbf{v}, t) & =\mathcal{N} \exp \left\{-\frac{m}{2 T}\left(\left[\frac{c_{1}(t) \mathbf{r}}{t_{s}}-c_{2}(t) \mathbf{v}\right]^{2} \mp \frac{t_{s}^{3}}{4 t_{0}^{3}}\left[\frac{c_{3}(t) \mathbf{r}}{t_{s}}-c_{4}(t) \mathbf{v}\right]^{2}\right)\right\} \\
& \left.\times \Theta\left(R_{0}-\left|c_{3}(t) \mathbf{r}-c_{4}(t) t_{s} \mathbf{v}\right|\right)\right) .
\end{aligned}
$$


The normalization constant $\mathcal{N}$ is chosen so that the distribution is normalized to unity at the initial time $t_{0}$.

$$
\int d^{3} r d^{3} v f^{ \pm}\left(\mathbf{r}, \mathbf{v}, t_{0}\right)=1
$$

Of course, this ensures that the distribution is normalized to unity for all later times, even when account is taken of the fact that the solution differs outside the expanding sphere.

For negative kaons the dimensionless functions of time $c_{i}(t)$ are given by

$$
\begin{aligned}
& \frac{c_{1}(t)}{t_{s}}=\frac{\pi}{4} \frac{t_{s}}{t_{0} t}\left[J_{2}\left(\sqrt{t_{s} / t_{0}}\right) N_{2}\left(\sqrt{t_{s} / t}\right)-N_{2}\left(\sqrt{t_{s} / t_{0}}\right) J_{2}\left(\sqrt{t_{s} / t}\right)\right] \\
& c_{2}(t)=\frac{\pi}{2} \sqrt{\frac{t_{s} t}{t_{0}^{2}}}\left[J_{2}\left(\sqrt{t_{s} / t_{0}}\right) N_{1}\left(\sqrt{t_{s} / t}\right)-N_{2}\left(\sqrt{t_{s} / t_{0}}\right) J_{1}\left(\sqrt{t_{s} / t}\right)\right] \\
& \frac{c_{3}(t)}{t_{s}}=\frac{\pi}{2} \sqrt{\frac{t_{0}}{t_{s} t^{2}}}\left[J_{1}\left(\sqrt{t_{s} / t_{0}}\right) N_{2}\left(\sqrt{t_{s} / t}\right)-N_{1}\left(\sqrt{t_{s} / t_{0}}\right) J_{2}\left(\sqrt{t_{s} / t}\right)\right] \\
& c_{4}(t)=\pi \sqrt{\frac{t_{0} t}{t_{s}^{2}}}\left[J_{1}\left(\sqrt{t_{s} / t_{0}}\right) N_{1}\left(\sqrt{t_{s} / t}\right)-N_{1}\left(\sqrt{t_{s} / t_{0}}\right) J_{1}\left(\sqrt{t_{s} / t}\right)\right] .
\end{aligned}
$$

For positive kaons the corresponding $c_{i}(t)$ are obtained by the substitution $t_{s} \rightarrow-t_{s}$ in the right-hand side of eqs. (15) and the identities

$$
\begin{aligned}
N_{n}(i x) & =i^{n+1} I_{n}(x)-\frac{2}{\pi}(-i)^{n} K_{n}(x), \\
J_{n}(i x) & =i^{n} I_{n}(x) .
\end{aligned}
$$

Generally the asymptotic momentum distribution is obtained by integrating over position and taking the limit $t \rightarrow \infty$. Once a kaon has crossed the surface of the expanding sphere it will see a time-independent potential, hence its energy will thereafter be conserved. Since a positive kaon will see a positive potential, its kinetic energy will not decrease, and neither will its velocity. Once a positive kaon has crossed the surface it will not come back. This will not be true for negative kaons; they will be discussed later. Due to spherical symmetry, the distribution is a function only of the magnitude of the three dimensional momentum vector. The coordinate integration can be performed analytically with the result for positive kaons being

$$
\begin{aligned}
& \frac{d^{3} N}{d^{3} p}=\pi \mathcal{N}\left(\frac{R_{0}}{\chi \delta}\right)^{3} \exp \left(-p^{2} / 2 m T \chi^{2}\right) \\
& \times\left\{\frac{\sqrt{\pi}}{2 i}\left[\Phi\left(\frac{p+\delta p_{C}}{i p_{C}}\right)-\Phi\left(\frac{p-\delta p_{C}}{i p_{C}}\right)\right] \exp \left(-p^{2} / p_{C}^{2}\right)+\exp \left(\delta^{2}\right) \frac{\sinh \left(2 \delta p / p_{C}\right)}{p / p_{C}}\right\}
\end{aligned}
$$

where $\Phi(x)$ is the error function and

$$
p_{C}^{2}=\frac{8 m T t_{0}}{t_{s}}\left(I_{2}^{2}-I_{1}^{2}\right) \frac{I_{1}^{2}}{I_{2}^{2}}
$$




$$
\begin{aligned}
\chi^{2} & =\frac{4 t_{0}}{t_{s}} I_{1}^{2} \\
\delta^{2} & =\frac{m v_{s}^{2} t_{s}}{8 T t_{0}}\left(\frac{I_{2}^{2}}{I_{1}^{2}}-1\right)
\end{aligned}
$$

where the Bessel functions are all evaluated at $\sqrt{t_{s} / t_{0}}$. Taking $Z=158, R_{0}=8 \mathrm{fm}, v_{s} / c$ $=0.4$, and $T=120 \mathrm{MeV}$, results in $t_{0}=20 \mathrm{fm} / \mathrm{c}, t_{s}=28.7 \mathrm{fm} / \mathrm{c}, p_{C}=1390 \mathrm{MeV} / \mathrm{c}, \chi$ $=1.19$, and $\delta=0.330$. It is important to remember that eq. (17) is valid only so long as the test particle (positive kaon) remains within the expanding sphere. This will be the case if its asymptotic speed is less than $v_{s}$.

To compute the distribution of positive kaons for asymptotic speeds greater than $v_{s}$ is straightforward due to the fact that once it has crossed the boundary of the expanding sphere, it sees a time independent potential so that its energy is thereafter conserved. The flux of particles crossing the surface at time $t$ is

$$
\left.\int d^{3} v \mathbf{v} \cdot \hat{\mathbf{r}} f^{+}(\mathbf{r}, \mathbf{v}, t)\right|_{r=v_{s} t}
$$

which is to be evaluated at $r=v_{s} t$. The energy distribution is obtained by multiplying by the surface area, inserting an energy conserving $\delta$ function, and integrating over time.

$$
\begin{aligned}
d N^{+} / d E & =\left.\int_{t_{0}}^{\infty} d t v_{s}^{2} t^{2} \int d \Omega_{\mathbf{r}} \int d^{3} v \mathbf{v} \cdot \hat{\mathbf{r}} f^{+}(\mathbf{r}, \mathbf{v}, t)\right|_{r=v_{s} t} \\
& \times \delta\left(E-p^{2} / 2 m-Z e^{2} / 4 \pi v_{s} t\right)
\end{aligned}
$$

The asymptotic momentum distribution is readily obtained from the energy distribution.

Figure 3 shows the $K^{+}$distributions for expanding fireballs with initial radius $8 \mathrm{fm}$, kaon temperature $120 \mathrm{MeV}$, and expansion velocities $v_{s} / c=0.2,0.4$ and 0.6 . For comparison the dotted curve shows the asymptotic distribution obtained from a static fireball. There is a discontinuity in the slope of the distributions at $E=\frac{1}{2} m v_{s}^{2}$. We have checked that the areas under all curves are 1 . The significant physics here is that the distribution remains finite at zero energy. The reason is that the charged fireball blows apart and leaves a finite number of low energy kaons behind. This cannot happen with a static fireball where a classical turning point exists. In the limit that the fireball expansion velocity is large compared to the thermal velocity of the kaons, the kaon distribution is hardly changed from the pure exponential form. The fireball expands so fast that it leaves the kaon distribution frozen to its initial shape. In the limit that the expansion velocity goes to zero, the static fireball result is recovered.

Now we turn to negative kaons. A negatively charged kaon can cross the fireball's surface if it has a sufficiently large radial velocity. After crossing the surface, it will slow down due to the attractive potential. It may even slow enough so that the surface passes it by! So the possibilities are three-fold: it always remains within the expanding sphere, it crosses the surface once and never comes back, or it crosses the surface twice so 
that asymptotically it remains within the expanding sphere. Kaons with an asymptotic energy greater than $\frac{1}{2} m v_{s}^{2}$ can be dealt with by the flux formula (19). Kaons with an asymptotic energy less than this cannot be handled analytically. This is ultimately due to the fact that the Coulomb trajectories cannot be expressed in terms of elementary functions of the time and so it is not possible to construct the phase space distribution outside the expanding sphere in a useful form. To handle this situation we have written some computer programs which calculate the trajectory of a kaon given its initial phase space position. One program was written in turbopascal with a screen display in real time which graphically shows the behaviors sketched above. A second program was written in fortran which connects the initial phase space point to the final energy (usually the asymptotic energy was determined at $100 t_{0}$ ).

Figure 4 shows the $K^{-}$distributions corresponding to the same fireball configurations as in figure 3. The solid curves show the result of the flux calculation for asymptotic energies greater than $\frac{1}{2} m v_{s}^{2}$. The histograms are the result of numerically computing the kaon trajectories for 720,000 initial phase space points. The initial radial position was incremented in $0.1 \mathrm{fm}$ bins, the initial speed was incremented in $0.01 \mathrm{c}$ bins, and the angle between the initial position and velocity vectors was incremented in $2^{\circ}$ bins. (Hence $80 \times 100 \times 90=720,000$.) The distribution is enhanced at small energies relative to the neutral kaons and to the positive kaons, but not as much as for a static fireball, shown in the figure by dotted curves. Another distinction is that there are no classically bound $K^{-}$, unlike the case of a static fireball.

The kaon data which originally attracted attention was for rather forward rapidities of 2.1 to 2.5 in contrast to the rapidity of the nucleus-nucleus center-of-mass frame of about 1.5. Plotted in the nucleus-nucleus center-of-mass frame the kinetic energies go no lower than $80 \mathrm{MeV}$ for $K^{-}$and $70 \mathrm{MeV}$ for $K^{+}$. It is problematic to compare against theoretically calculated spectra for kinetic energies greater than about $100 \mathrm{MeV}$ when a nonrelativistic approximation has been made. In addition, the selection criterion was for the $10 \%$ most central collisions, corresponding to the $30 \%$ most central impact parameters. These impact parameters are really too large to focus on truly central collisions of equal mass nuclei.

In conclusion we have shown the importance of the Coulomb force on charged kaons in central collisions of large nuclei at high energies. We have shown how the expansion of the fireball affects the kaon distributions in a quantitative way. Comparison to data at kinetic energies greater than about $100 \mathrm{MeV}$ probably requires a relativistic treatment which we are now working on. By varying the initial phase space distribution of the kaons by invoking some component of flow, for example, and comparing with data should yield quantitative information on the dynamics of kaons and heavy ion collisions. 


\section{Acknowledgements}

The authors wish to thank J. Stachel and R. Lacasse for discussing the data of E814/E877. J. K. thanks B. Bayman for a turbopascal program which solves Newton's equation for the motion of a planet. A. A. thanks the Institute for Nuclear Theory at the University of Washington for its hospitality and the Department of Energy for its support there during the completion of this work. This work was supported by the US National Science Foundation under grant NSF PHY94-21309 and by the US Department of Energy under grant DE-FG02-87ER40328.

\section{References}

[1] J. Stachel et al. (E814 Collaboration), Proceedings of Quark Matter '93, Nucl. Phys. A566, 183c (1994); J. Barrette et al. (E877 Collaboration), Proceedings of Quark Matter '95, Nucl. Phys. A590, 259c (1995).

[2] R. Lacasse et al. (E877 Collaboration), Proceedings of Quark Matter '96, in press.

[3] M. Gyulassy and S. K. Kauffmann, Nucl. Phys. A362, 503 (1981).

[4] L. P. Csernai and J. Kapusta, Phys. Rev. D 46, 1379 (1992).

[5] Y. Pang, T. J. Schlagel and S. H. Kahana, Nucl. Phys. A544, 435c (1992).

[6] H. Sorge, H.Stöcker and W. Greiner, Nucl. Phys. A498, 507c (1989). 


\section{Figure Captions}

Fig. 1: Gamow factor momentum distributions $d^{3} N / d^{3} p$ as a function of kinetic energy $E$ for a non-expanding fireball with $T=120 \mathrm{MeV}$ and $Z=158$. Also shown is the corresponding neutral distribution.

Fig. 2: Momentum distributions $d^{3} N / d^{3} p$ as a function of kinetic energy $E$ for a nonexpanding fireball with $R_{0}=8 \mathrm{fm}, T=120 \mathrm{MeV}, Z=158$. The sudden drop in the $K^{+}$ distribution signals the proximity of $E$ to $E_{\min } \equiv Z e^{2} / 4 \pi R_{0}$, the classical turning point. Compare this to figure 1.

Fig. 3: $K^{+}$momentum distributions $d^{3} N / d^{3} p$ as a function of kinetic energy $E$ for a uniformly expanding fireball with $R_{0}=8 \mathrm{fm}, T=120 \mathrm{MeV}, Z=158$ and various values of the expansion velocity $v_{s}$ indicated with solid lines. Also shown is the corresponding result for a static fireball $v_{s}=0$ indicated with dotted lines.

Fig. 4: $K^{-}$momentum distributions $d^{3} N / d^{3} p$ as a function of kinetic energy $E$ for a uniformly expanding fireball with $R_{0}=8 \mathrm{fm}, T=120 \mathrm{MeV}, Z=158$ and various values of the expansion velocity $v_{s}$. The solid lines starting at $E_{s}=\frac{1}{2} m v_{s}^{2}$ are obtained from the flux across the fireball's surface. The histograms are obtained by solving the classical equations of motion numerically for 720,000 starting points in phase space. Also shown is the corresponding result for a static fireball $v_{s}=0$ indicated with dotted lines. 
Fig. 3a

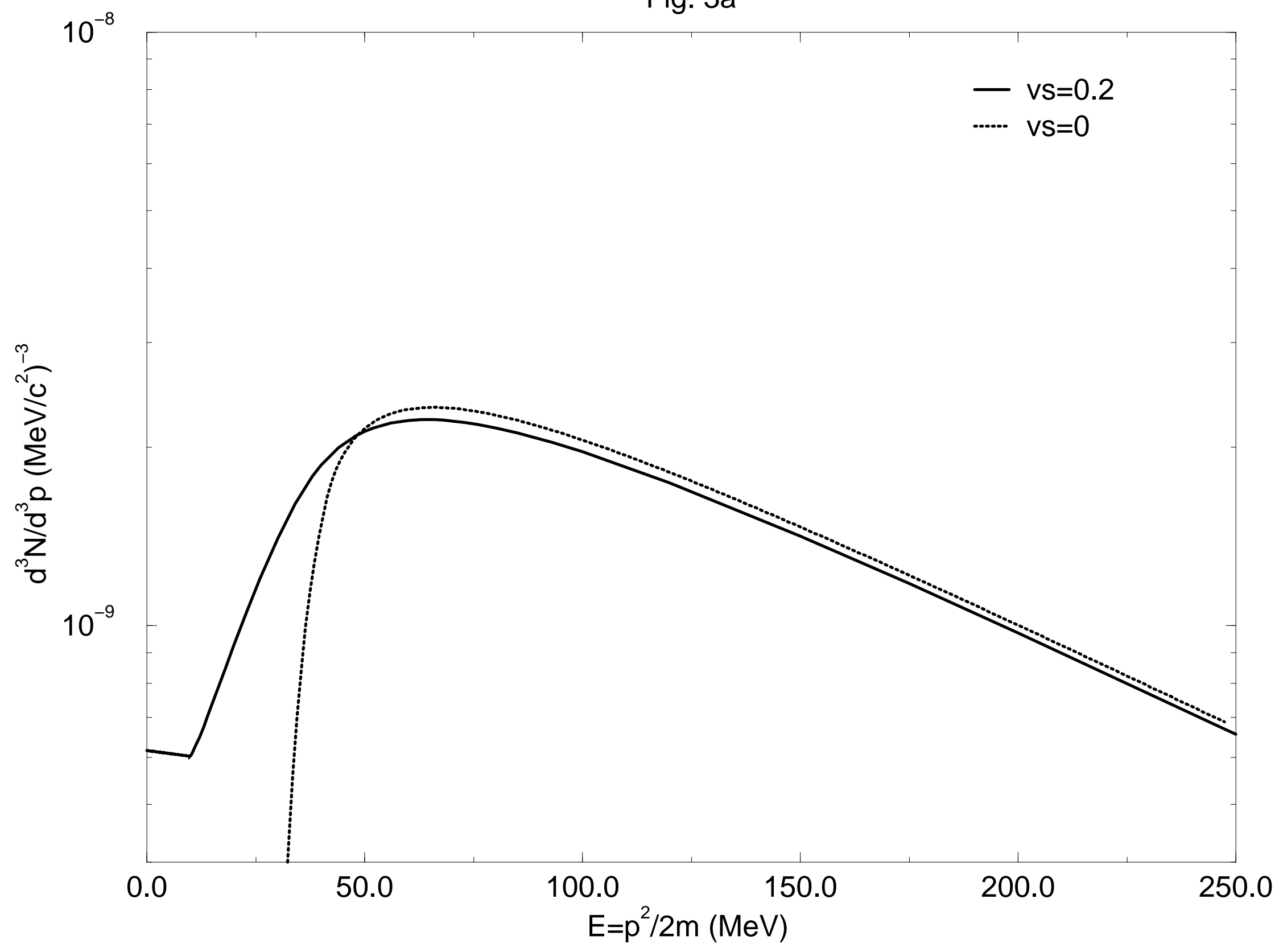


Fig. $3 b$

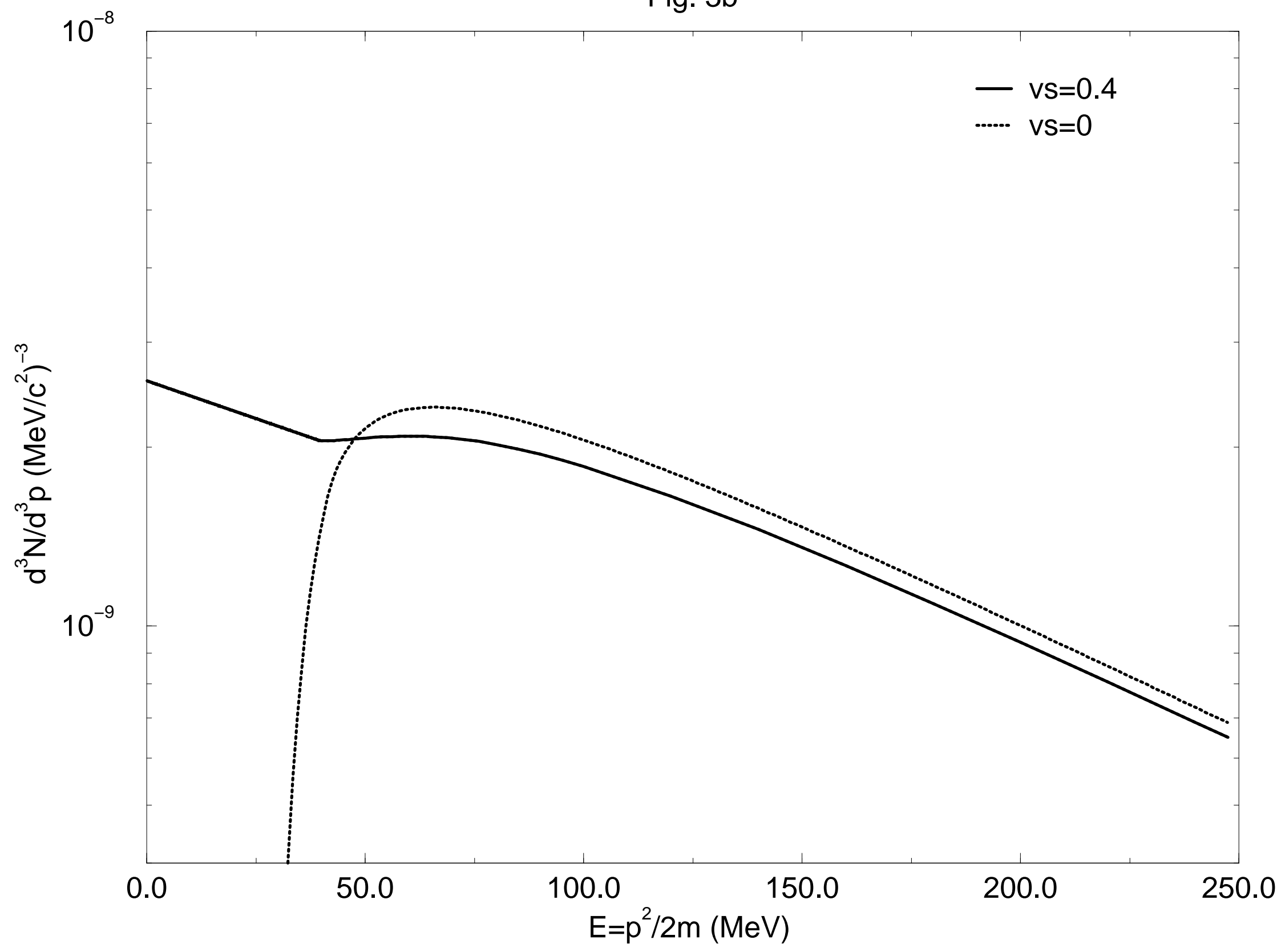


Fig. 3c

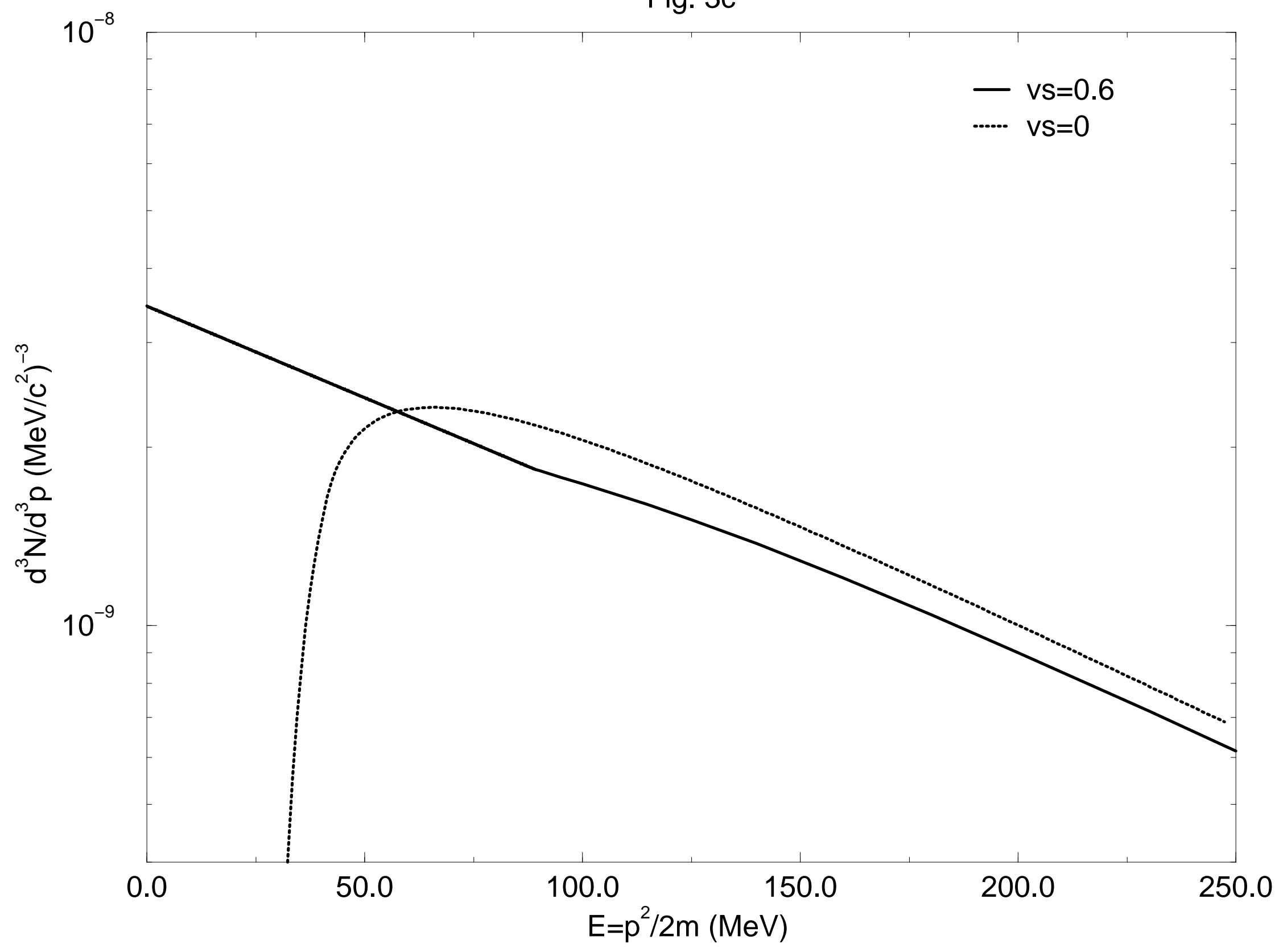


Fig. $4 a$

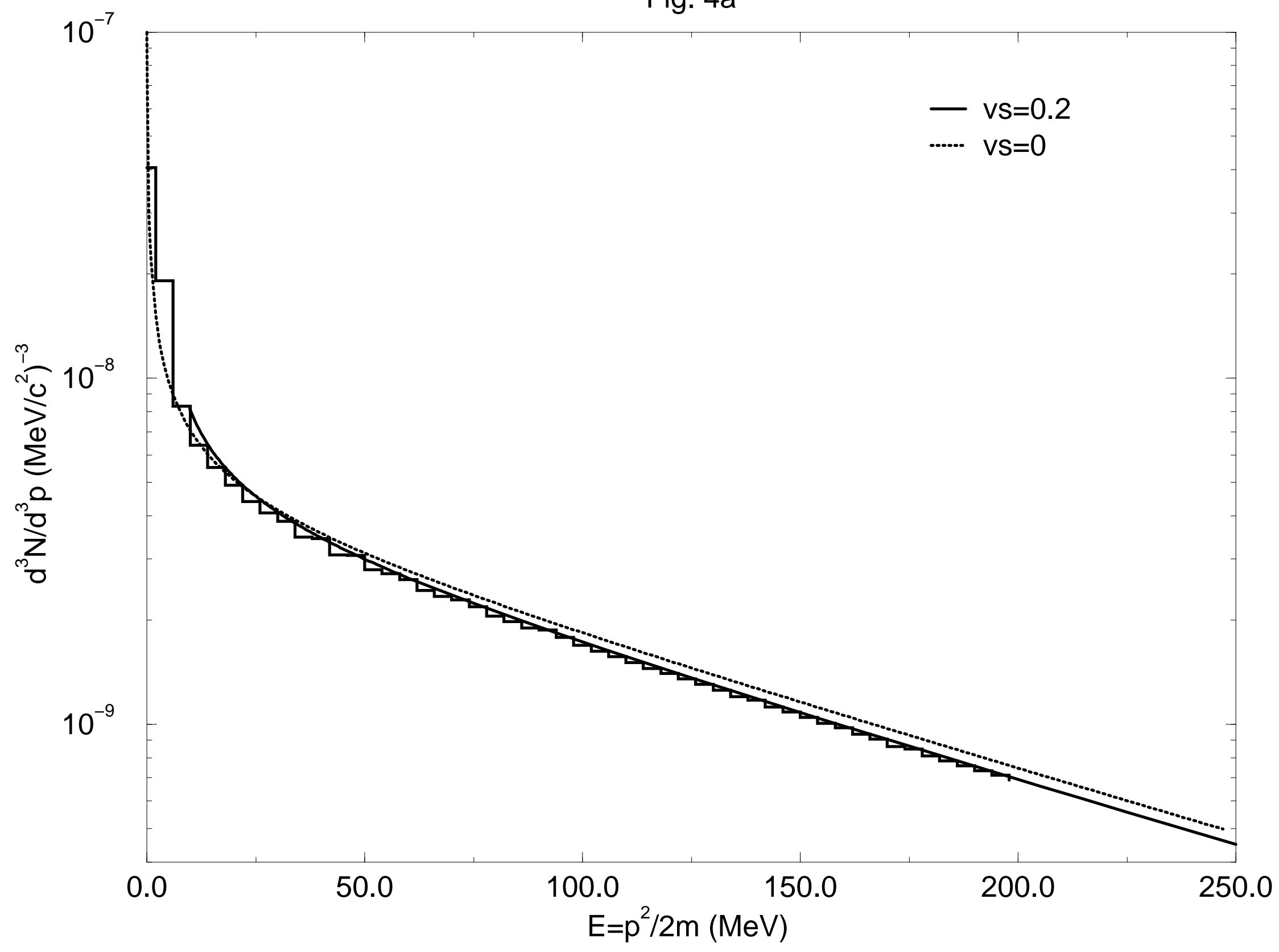


Fig. 4b

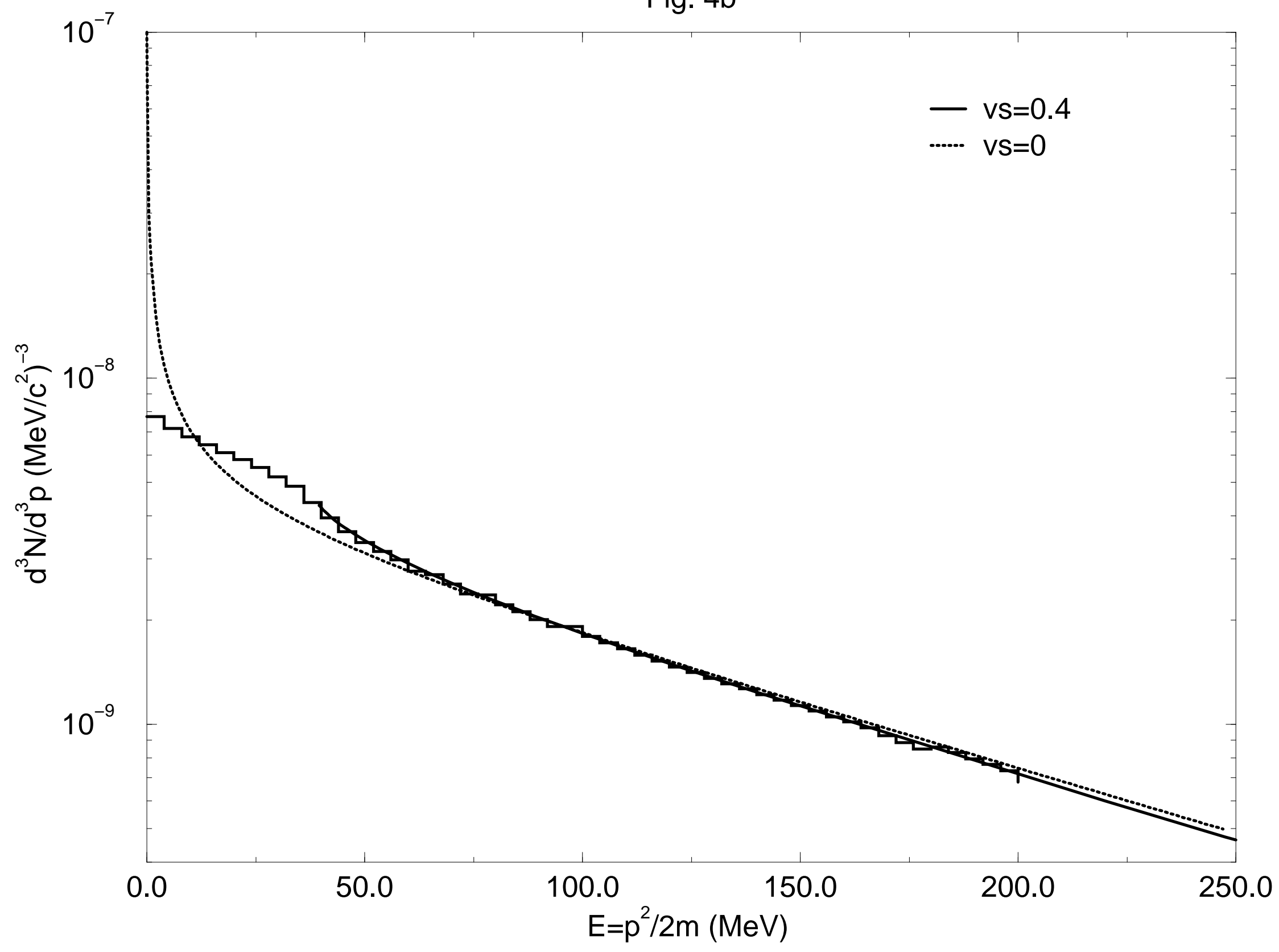


Fig. 4c

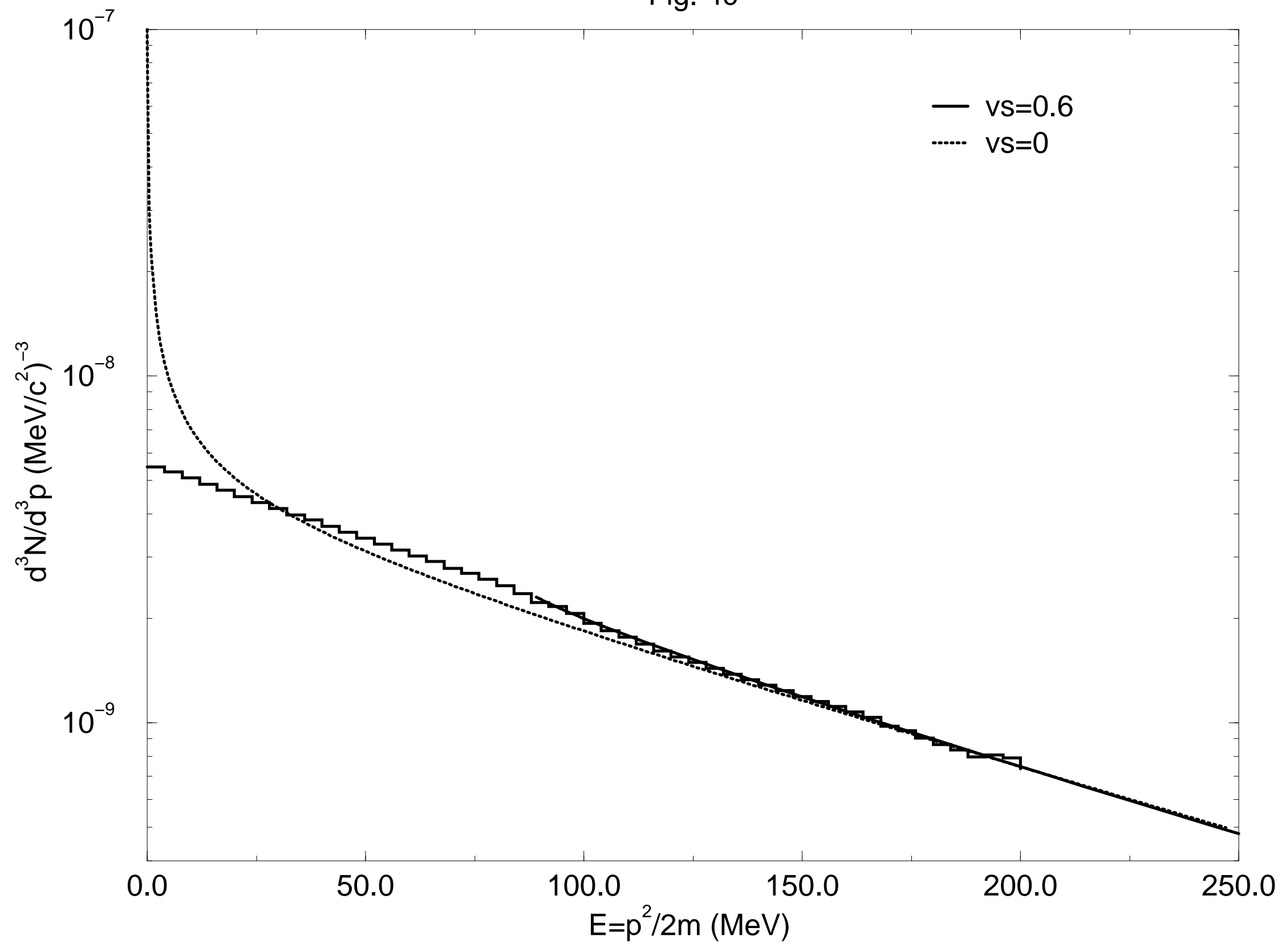


Fig. 1

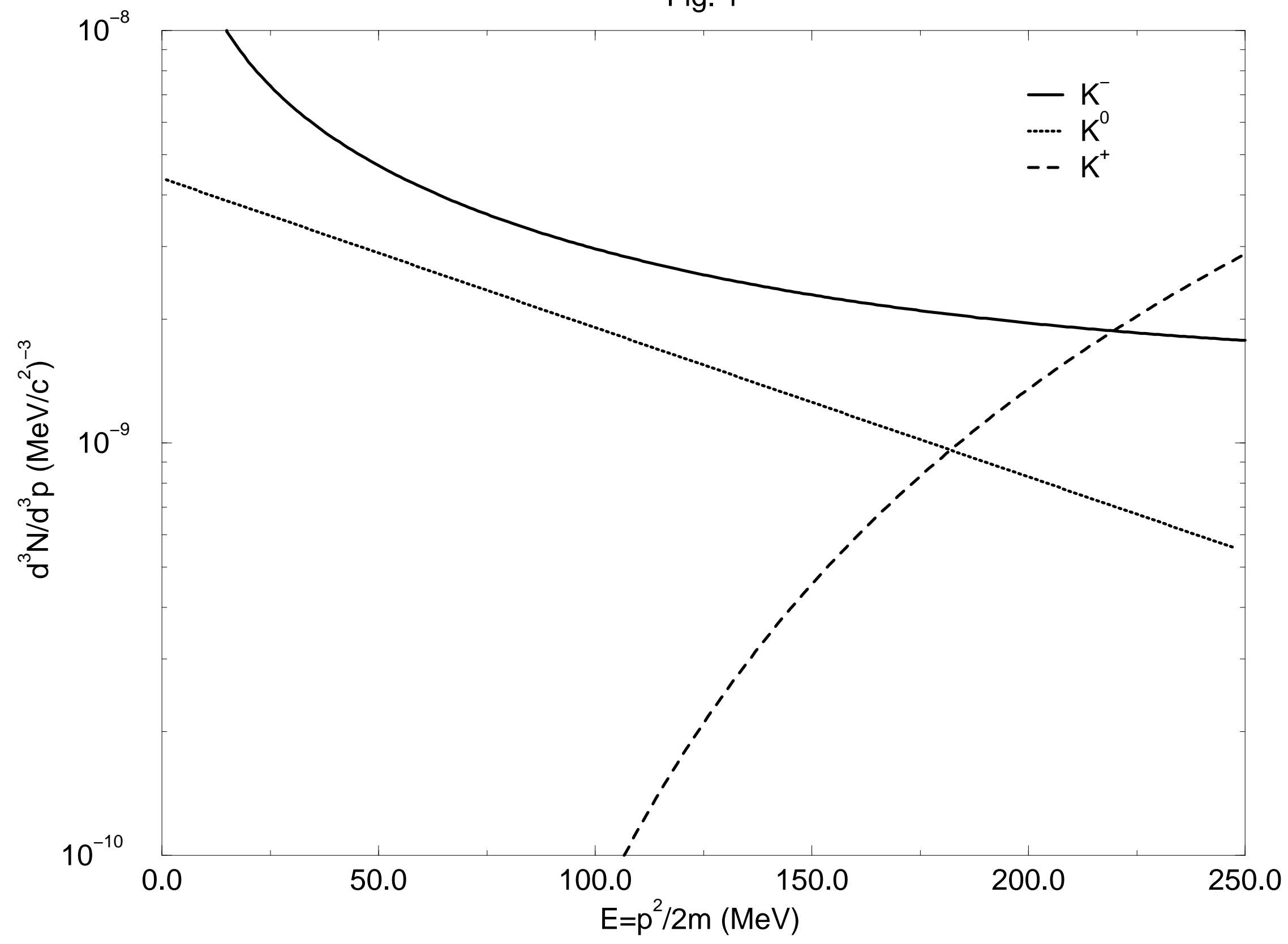


Fig. 2

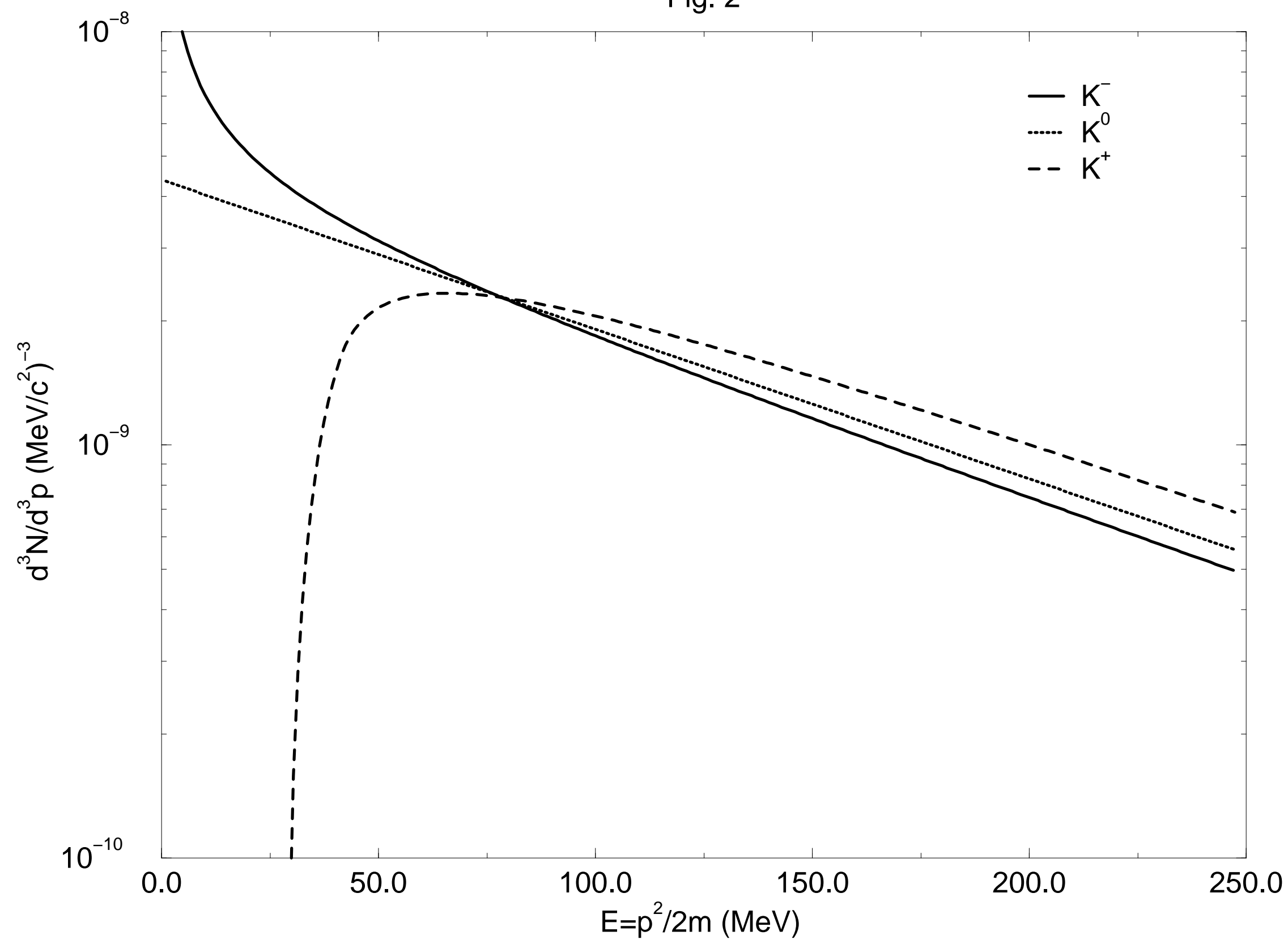

